\title{
Use of a new LC-MS method for the determination of pyrrolizidine alkaloids in feeds
}

\author{
Ewelina Kowalczyk, Krzysztof Kwiatek \\ Department of Hygiene of Animal Feedingstuffs \\ National Veterinary Research Institute, 24-100 Pulawy, Poland \\ ewelina.kowalczyk@piwet.pulawy.pl
}

Received: February 8, 2018 Accepted: June 8, 2018

\begin{abstract}
Introduction: Pyrrolizidine alkaloids (PAs) are secondary metabolites produced by many plant species. Due to their toxicity PAs can pose a risk to human and animal health. To detect the toxic compounds in feed materials a sensitive method based on liquid chromatography coupled with mass spectrometry has been developed. Material and Methods: PAs were extracted with sulphuric acid and purified with cation exchange cartridges. A newly developed solvent mixture consisting of ethyl acetate, methanol, acetonitrile, ammonia, and triethylamine was used to wash alkaloids from the cartridges. After evaporation the residues were reconstituted in water and methanol mixture and subjected to LC-MS analysis. Results: The developed method was validated according to SANTE/11945/2015 guidelines. The recovery was from $84.1 \%$ to $112.9 \%$, the repeatability ranged from $3.0 \%$ to $13.6 \%$, and the reproducibility was from $4.8 \%$ to $18.9 \%$. Conclusions: A sensitive and selective method for determination of PAs in feed materials has been developed and validated. All evaluated validation parameters were in accordance with EU Reference Laboratories document no. SANTE/11945/2015. Almost 41\% of the analysed feed samples were positive for the presence of at least one PA.
\end{abstract}

Keywords: feed, pyrrolizidine alkaloids, LC-MS.

\section{Introduction}

Pyrrolizidine alkaloids (PAs) are synthesised by plants as their secondary metabolites and are considered to be one of the most widespread toxins of natural origin $(1,23)$. PAs have gained attention in the recent years due to their hepatotoxic, carcinogenic, genotoxic, and pneumotoxic properties (17).

More than 6,000 plant species produce alkaloids and more than 660 PAs have been identified so far (5, 15). PA-producing plants are introduced or are native species which are considered invasive and noxious weeds. They often infest open ranges and fields leading to contamination of crops and feeds $(12,30)$.

PAs are esters of amino alcohols, consisting of two basic structure elements: a pyrrolizidine-derived moiety necine and different nono- or dicarboxylic acids. The necine base consists of two fused fivemembered rings with a nitrogen atom at the bridgehead, which can be saturated or contain a double bond in the 1,2-position (15). PAs occur as free base/tertiary forms and as $N$-oxides which are characterised by different reactivity and solubility leading to different toxicity (3). Neither form is significantly toxic to humans or animals per se. Their hazard arises when they are converted into pyrrolic metabolites in the liver. These highly reactive electrophilic alkylating agents are capable of binding strongly to nucleophilic centres in tissues or crosslinking DNA, leading to hepatotoxicity or carcinogenicity (14).

Cases of intoxication have been noted worldwide and toxicity effects of some PAs have been well documented in numerous publications $(10,13,16,24)$. Besides acute intoxications, pyrrolizidine alkaloids are considered to cause delayed progressive chronic effects, including hepatic cirrhosis following even short-term low-level exposure (14). Chronically poisoned animals often do not show any clinical signs and their serum biochemistry may be normal for several months or even years after PAs ingestion. Nevertheless, the lasting hepatocellular damage leads 
to increased hepatocyte death with subsequent inflammation, fibrosis, and ultimately cirrhosis (30). In an analogous way in humans, even single dietary exposure to dehydro PAs can lead to silently progressing chronic diseases which are unlikely to be attributed to dehydro PAs in food (12).

Animals are exposed to PAs mainly through feed contaminated with plants containing these compounds, though due to the bitter taste, animals usually avoid direct consumption of PA-producing plants $(2,15)$. It has also been proved that PAs can be transferred to food of animal origin such as milk, eggs, and meat, if animals consume contaminated feed $(8,19,27)$.

Only a few studies on the contamination of feed materials with PAs have been published hitherto $(2,18$, 20, 25). The problem of PA-contaminated feeds originating from Polish territory has not been well recognised. Only one paper on this subject has been published (21). In the previous study, PAs were determined with the gas chromatography mass spectrometry method (GC-MS) using the sum parameter approach. This approach allows PAs determination without the necessity of having analytical standards of all the particular PAs. However, the drawback of this approach is that 1,2-unsaturated alkaloids are reduced to their common base structures retronecine and heliotridine, and all information concerning the whole structure is lost. Where that information retained, it could lead to indication which of the alkaloids are present in the analysed material. For this reason, a new liquid chromatography method for determination of selected alkaloids has been developed.

Taking into consideration results reported by other authors $(2,18,20,25)$, the European Food Safety Authority (EFSA) recommendations, and PAproducing plants occurring in Poland, the following alkaloids were selected for the study: senecionine-type PAs including jacobine, retrorsine, senecionine, and seneciphylline; lycopsamine-type PAs including lycopsamine, intermedine, and echimidine; heliotrinetype PAs including heliotrine and lasiocarpine; and senkirkine (15). The final determined concentration of PAs also includes the $N$-oxides contribution, as they are reduced with zinc dust and are measured as free base form.

\section{Material and Methods}

Chemicals and reagents. Sulphuric acid (95\%) was from Chempur (Poland), 25\% ammonia solution was from POCH (Poland), and formic acid and zinc dust were from Sigma-Aldrich (USA). Ethyl acetate and triethylamine were from Merck (Germany), and methanol and acetonitrile were from J.T. Baker (the Netherlands). Water was purified with the Milli-Q water purification system (Millipore, USA). Analytical standards of intermedine, lycopsamine, jacobine, retrorsine, heliotrine, seneciphylline, senecionine, echimidine, senkirkine, and lasiocarpine were purchased from PhytoLab (Germany). Solid phase extraction (SPE) Strata SCX and polymeric Strata XC cartridges were supplied by Phenomenex (USA). Oasis MCX mixed-mode polymeric cartridges were from Waters (USA) and Bond Elut Plexa PCX and HF Bond Elut-SCX cartridges from Agilent (USA). The cartridges were of $500 \mathrm{mg}$ bed weight and $6 \mathrm{~mL}$ volume.

Standard solutions. Stock standard solutions of intermedine, lycopsamine, jacobine, retrorsine, heliotrine, seneciphylline, senecionine, echimidine, senkirkine, and lasiocarpine were prepared at a concentration of $1 \mathrm{mg} \mathrm{mL}^{-1}$ in methanol and stored at $-18^{\circ} \mathrm{C}$. A mixed standard solution of $1 \mu \mathrm{g} \mathrm{mL}^{-1}$ was prepared by mixing the appropriate volume of each stock standard solution and subsequent serial dilution. The mixed standard solution was stored between 2 and $4^{\circ} \mathrm{C}$.

Feed samples. Analysed feed materials included grass and alfalfa silage (27 and 1 sample, respectively) and hay (4 samples). All analysed samples were available from earlier microbiological studies.

Sample preparation. Silage was dried in an oven and then ground and homogenised as well as hay. Homogenised feed material (5 g) was weighed into $50 \mathrm{~mL}$ polypropylene tubes and $40 \mathrm{~mL}$ of $0.05 \mathrm{M}$ sulphuric acid was added. The samples were extracted for $2 \mathrm{~h}$ on a horizontal shaker. After extraction, the samples were centrifuged and filtered through cellulose filters. About $1 \mathrm{~g}$ of zinc dust was added to filtered extracts to reduce $\mathrm{N}$-oxides and the samples were left overnight. On the next day, the samples were slowly shaken for $0.5 \mathrm{~h}$, subsequently centrifuged $(4,000 \mathrm{~g}$, $10 \mathrm{~min}$ ), and $15 \mathrm{~mL}$ of the extract was purified with SPE. Strata SCX cartridges were preconditioned with $9 \mathrm{~mL}$ of methanol and $9 \mathrm{~mL}$ of $0.05 \mathrm{M} \mathrm{H}_{2} \mathrm{SO}_{4}$ acid. After sample application, the cartridges were washed with $12 \mathrm{~mL}$ of water and $12 \mathrm{~mL}$ of methanol and vacuum dried for $2 \mathrm{~min}$, then $6 \mathrm{~mL}$ of ethyl acetate was added. For the elution of PAs, $12 \mathrm{~mL}$ of solvent mixture consisting of ethyl acetate, methanol, acetonitrile, ammonia, and triethylamine (8:1:1:0.1:0.1 v/v) was used. After evaporation at $40^{\circ} \mathrm{C}$ in a nitrogen stream the residues were reconstituted in $0.2 \mathrm{~mL}$ of water and $0.2 \mathrm{~mL}$ of methanol and passed through $0.2 \mu \mathrm{m}$ PVDF syringe filters into chromatographic vials.

Instrumental parameters. HP 1200 Series separation modules from Agilent Technologies (USA) were used for the analysis. These modules consisted of a degasser system, binary pump, automatic injector, and column thermostat. A single quadrupole mass spectrometry detector (6140 Agilent Technologies) was also employed. The separation of the alkaloids was carried out on a Gemini $3 \mu \mathrm{m}$ NX-C18, $150 \mathrm{~mm} \times 4.6 \mathrm{~mm}$ column (Phenomenex, USA) coupled with $\mathrm{C} 18$ guard column (Phenomenex). The column was thermostatted 
at $30^{\circ} \mathrm{C}$. The mobile phase containing $0.2 \%$ formic acid in water $(\mathrm{A})$ and a mixture of methanol and acetonitrile $(1: 1, \mathrm{v} / \mathrm{v})(\mathrm{B})$ was used in a gradient mode as follows: 0-2 min, 5.5\% B; 2-8 min, $12 \%$ B; $8-11 \mathrm{~min}, 20 \% \mathrm{~B}$; 11-12 $\mathrm{min}, 30 \% \mathrm{~B} ; 12-15 \mathrm{~min}, 40 \% \mathrm{~B} ; 17-16 \mathrm{~min}$, $70 \% \mathrm{~B} ; 16-17 \mathrm{~min}, 85 \% \mathrm{~B}$; and $17-23 \mathrm{~min}, 5.5 \% \mathrm{~B}$.

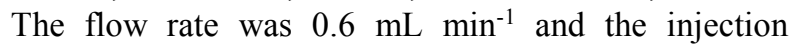
volume was $5 \mu \mathrm{L}$. Electrospray ionisation (ESI) was set in a positive mode, the capillary voltage was set at $2,000 \mathrm{~V}$, nebulizer pressure was $35 \mathrm{psi}$, and drying gas flow and temperature were $11.0 \mathrm{~L} \mathrm{~min}^{-1}$ and $300^{\circ} \mathrm{C}$, respectively. Fragmentor voltage was set at $100 \mathrm{~V}$ for all monitored alkaloids. Selected ion monitoring was used for the detection, and the protonated molecular $(\mathrm{M}+\mathrm{H})^{+}$ions $(\mathrm{m} / \mathrm{z})$ monitored are listed in Table 1.

Identification and quantification. Identification was made by comparison with the relevant reference standard by the retention time and the protonated molecular ion $(\mathrm{M}+\mathrm{H})^{+}$. Quantification was achieved using calibration curves prepared by adding the mixed reference standard solution to blank matrix in appropriate amounts before the extraction procedure. Calibration curves were constructed by plotting the peak area versus the alkaloid concentrations.

Validation of the method. In-house validation was conducted according to SANTE/11945/2015 guidelines (28). Parameters such as linearity, recovery, repeatability, reproducibility, specificity, limit of quantification (LOQ), matrix effect, robustness, and PA stability were evaluated. Tested feed with no determined content of the target compounds was used as a blank matrix.

Linearity and selectivity. The method's linearity was evaluated using matrix calibration curves. All curves were composed by blank samples fortified with the standards before the extraction procedure and analysed in triplicate. Feed samples were spiked with appropriate concentrations corresponding to $0,5,10$, 20, 30, 50, and $100 \mu \mathrm{g} \mathrm{kg}^{-1}$.

To determine the selectivity of the method a set of blank feed samples was analysed in order to check the possible presence of endogenous interferences in retention times of the monitored alkaloids.
Recovery and precision. Recovery and precision were determined by the analysis of feed blanks spiked at three different concentrations, corresponding to 5, 20, and $100 \mu \mathrm{g} \mathrm{kg}^{-1}$ (a set of six samples for each level). The samples were analysed under the same conditions. The repeatability was expressed as relative standard deviation of the results, RSD (\%). To determine the reproducibility, also expressed as RSD $(\%)$, other two sets of feed samples were spiked at 5 , 20 , and $100 \mu \mathrm{g} \mathrm{kg}^{-1}$, and analysed on different days with the same instrument.

Quantification limit and matrix effect. According to the SANTE document (28) the lowest spike level meeting the method performance criteria for trueness and precision was assumed to be the limit of quantification. Matrix effects are generally recognised as a suppression or enhancement of the analytical signal due to co-eluting matrix components (9). This effect was evaluated by comparison of the slopes of matrix matched calibration curves with slopes of calibration curves in a solvent.

Robustness and stability test. The Youden procedure was used to determine the robustness of the method. Blank feeds spiked at the concentration of $20 \mu \mathrm{g} \mathrm{kg}^{-1}$ were analysed in order to assess the influence of seven parameters which were slightly changed. The effects of change of percentage of sulphuric acid $(0.05 \mathrm{M}, 0.1 \mathrm{M})$, volume of elution mixture $(12 \mathrm{~mL}, 10 \mathrm{~mL})$, evaporation temperature $\left(40^{\circ} \mathrm{C}, 45^{\circ} \mathrm{C}\right)$, percentage of formic acid in the mobile phase $(0.2 \%, 0.18 \%)$, injection volume $(5 \mu \mathrm{L}, 4.5 \mu \mathrm{L})$, thermostat temperature $\left(30^{\circ} \mathrm{C}, 27^{\circ} \mathrm{C}\right)$, and flow rate $\left(0.6 \mathrm{~mL} \mathrm{m^{-1 }}, 0.63 \mathrm{~mL} \mathrm{~min}^{-1}\right)$ were evaluated. Student's $t$-test was used to determine the impact of changes in individual parameters on the results of the analysis.

A stability test was performed on SPE purified extracts. Blank feeds were contaminated at the level of $20 \mu \mathrm{g} \mathrm{kg}^{-1}$ before the extraction procedure. Dry extracts were stored at $-18^{\circ} \mathrm{C}, 4^{\circ} \mathrm{C}$, and $20^{\circ} \mathrm{C}$. The extracts were subsequently analysed on days 1, 2, 4, 8, 14, and 30 .

Table 1. Selected $(\mathrm{m} / \mathrm{z})$ ions and retention times of the monitored alkaloids. Determination coefficients obtained for matrix calibration curves in a concentration range corresponding to $0-100 \mu \mathrm{g} \mathrm{kg}^{-1}$ for all analysed alkaloids, and results of matrix effect evaluation. PA pyrrolizidine alkaloid, $\mathrm{RT}$ - retention time, $\mathrm{R}^{2}$ - coefficient of determination, $\mathrm{ME}$ - matrix effect

\begin{tabular}{lllll}
\hline $\mathrm{PA}$ & $\mathrm{m} / \mathrm{z}$ & $\mathrm{RT}$ & $\mathrm{R}^{2}$ & $\mathrm{ME}(\%)$ \\
\hline Intermedine & 300.1 & 6.14 & 0.999 & 99.9 \\
Lycopsamine & 300.0 & 6.37 & 0.999 & 108.6 \\
Jacobine & 352.1 & 6.88 & 0.999 & 93.7 \\
Retrorsine & 352.1 & 8.29 & 0.996 & 98.3 \\
Heliotrine & 314.1 & 8.58 & 0.999 & 102.2 \\
Seneciphylline & 334.1 & 9.11 & 0.998 & 98.9 \\
Senecionine & 336.1 & 11.09 & 0.998 & 100.3 \\
Echimidine & 398.0 & 13.11 & 0.997 & 100.6 \\
Senkirkine & 366.1 & 13.37 & 0.997 & 89.9 \\
Lasiocarpine & 412.1 & 15.03 & 0.995 & 101.4 \\
\hline
\end{tabular}




\section{Results}

Validation results. The developed method was validated in-house according to SANTE/11945/2015 guidelines (28). Matrix calibration curves were linear in the concentration range corresponding to $0-100 \mu \mathrm{g} \mathrm{kg}^{-1}$ for all analysed alkaloids. The linearity was confirmed as coefficients of determination were higher than 0.99 for all monitored PAs (Table 1).

All validation parameters (Table 2) were determined on the basis of the analysis of sets of blank feed samples spiked at the levels corresponding to concentrations 5, 20, and $100 \mu \mathrm{g} \mathrm{kg}^{-1}$. The recovery was from $84.1 \%$ to $112.9 \%$. The repeatability expressed as RSD (\%) ranged from $3.0 \%$ to $13.6 \%$, and reproducibility was from $4.8 \%$ to $18.9 \%$.

The robustness test revealed that chosen variables do not affect the analysis as the calculated standard deviation was not significantly higher than the standard deviation of the method carried out under withinlaboratory reproducibility conditions. The results were also compared with the use of Student's $t$-test to determine the impact of changes in individual parameters on the result of the analysis and no significant statistical differences were determined. Only a small retention time shift was observed when the flow rate was changed to $0.63 \mathrm{~mL} \mathrm{~min}{ }^{-1}$, or the thermostat temperature was decreased to $27^{\circ} \mathrm{C}$.

On the basis of the performed stability test, it can be stated that extracts of feed samples can be stored at $-18^{\circ} \mathrm{C}$ and at $4^{\circ} \mathrm{C}$ for about a week without a significant change in PAs concentrations (Fig. 1, data shown only for $-18^{\circ} \mathrm{C}$ ).

The method is selective as no interfering peaks were determined in the retention times of the monitored alkaloids (Fig. 2c). The LOQ was established at $5 \mu \mathrm{g} \mathrm{kg}^{-1}$ for the individual alkaloid, as it was the lowest validation level meeting the criteria of SANTE document for trueness and precision.

The matrix effect was in the range $\pm 20 \%$ (Table 1 ) for all compounds which complies with the SANTE document.

Real sample application. The developed method was applied to the analysis of 32 feed samples. Analysed feed materials included grass and alfalfa silage and hay. All analysed samples were available from earlier microbiological studies.

Among 32 analysed feed samples, 13 (40.6\%) contained at least one of the monitored PAs (2 positive hay samples and 11 positive silage samples). In two samples PAs concentrations were below LOQ value (Fig. 3).

Table 2. Validation parameters evaluated for ten alkaloids in feed matrix

\begin{tabular}{|c|c|c|c|c|c|c|c|c|c|}
\hline \multirow[b]{3}{*}{ PA } & \multicolumn{3}{|c|}{ Concentration level $\left(\mu \mathrm{g} \mathrm{kg}^{-1}\right)$} & \multicolumn{3}{|c|}{ Concentration level $\left(\mu \mathrm{g} \mathrm{kg}^{-1}\right)$} & \multicolumn{3}{|c|}{ Concentration level $\left(\mu \mathrm{g} \mathrm{kg}^{-1}\right)$} \\
\hline & 5 & 20 & 100 & 5 & 20 & 100 & 5 & 20 & 100 \\
\hline & \multicolumn{3}{|c|}{ Recovery $(\%)$} & \multicolumn{3}{|c|}{ Repeatability RSD (\%) } & \multicolumn{3}{|c|}{ Reproducibility RSD (\%) } \\
\hline Intermedine & 99.5 & 99.1 & 89.9 & 11.7 & 13.4 & 11.2 & 18.4 & 16.8 & 13.9 \\
\hline Lycopsamine & 89.1 & 97.9 & 87.6 & 9.6 & 11.6 & 12.8 & 18.3 & 10.6 & 16.8 \\
\hline Jacobine & 99.8 & 102.5 & 92.3 & 9.3 & 13.6 & 9.5 & 10.8 & 13.7 & 9.8 \\
\hline Retrorsine & 112.9 & 84.1 & 97.1 & 10.1 & 10.9 & 4.0 & 16.3 & 18.9 & 4.8 \\
\hline Heliotrine & 100.5 & 93.9 & 92.9 & 5.7 & 11.8 & 5.2 & 16.3 & 14.1 & 5.3 \\
\hline Seneciphylline & 102.3 & 100.2 & 97.1 & 9.5 & 12.2 & 4.3 & 18.8 & 12.7 & 5.2 \\
\hline Senecionine & 100.6 & 104.6 & 94.7 & 8.7 & 12.9 & 4.5 & 14.6 & 13.4 & 5.5 \\
\hline Echimidine & 110.8 & 109.5 & 95.0 & 7.4 & 8.9 & 7.0 & 13.9 & 11.8 & 5.5 \\
\hline Senkirkine & 94.2 & 106.4 & 97.5 & 3.0 & 5.6 & 5.9 & 18.0 & 9.9 & 8.0 \\
\hline Lasiocarpine & 100.5 & 99.4 & 97.2 & 6.0 & 7.9 & 3.8 & 11.6 & 15.1 & 6.3 \\
\hline
\end{tabular}

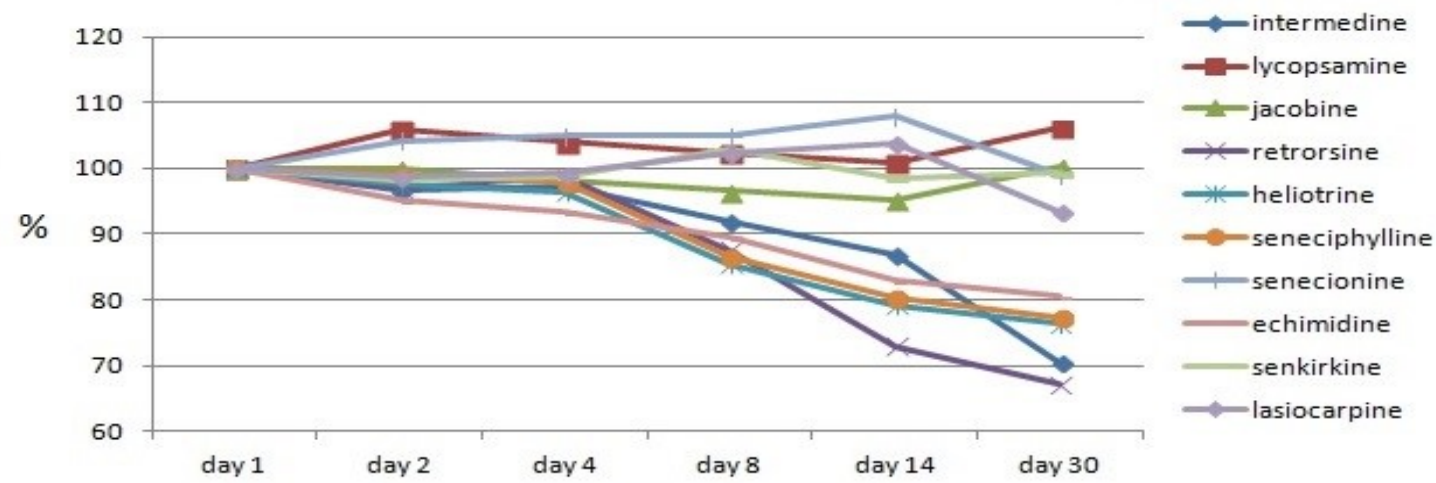

Fig. 1. Stability of ten PAs in dry SPE-purified extracts of feed matrix, stored at $-18^{\circ} \mathrm{C}$ 

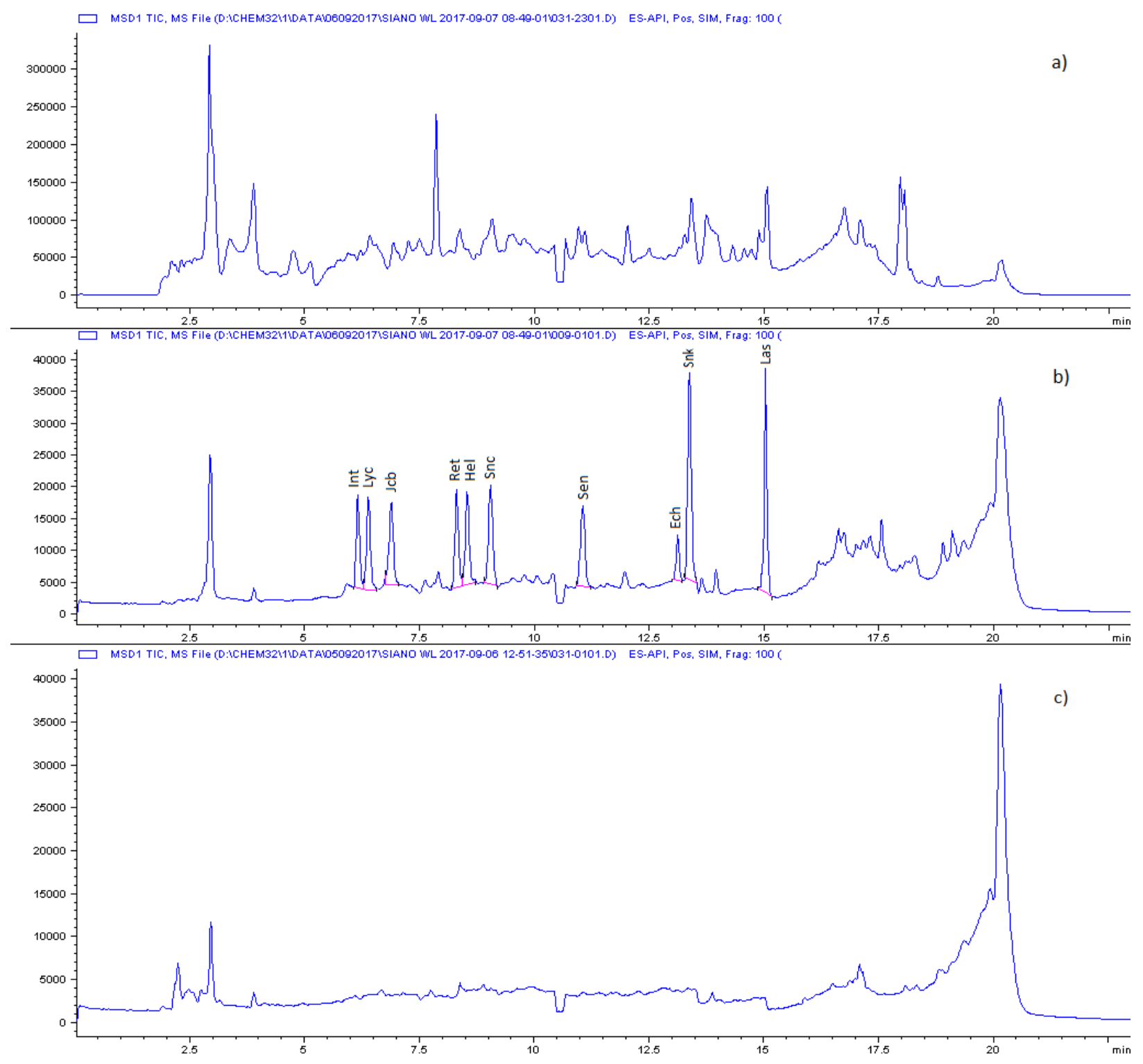

Fig. 2. SIM chromatograms obtained for: a) 3\% ammonia in methanol SPE elution (PAs concentration $20 \mu \mathrm{g} \mathrm{kg}^{-1}$ ); b) new solvent mixture consisting of ethyl acetate, methanol, acetonitrile, ammonia, and triethylamine (8:1:1:0.1:0.1, v/v) SPE elution (PAs concentration $5 \mu \mathrm{gg}^{-1}$ ); c) blank feed sample. Intermedine (Int), lycopsamine (Lyc), jacobine (Jcb), retrorsine (Ret), heliotrine (Hel), seneciphylline (Snc), senecionine (Sen), echimidine (Ech), senkirkine (Skn), and lasiocarpine (Las)

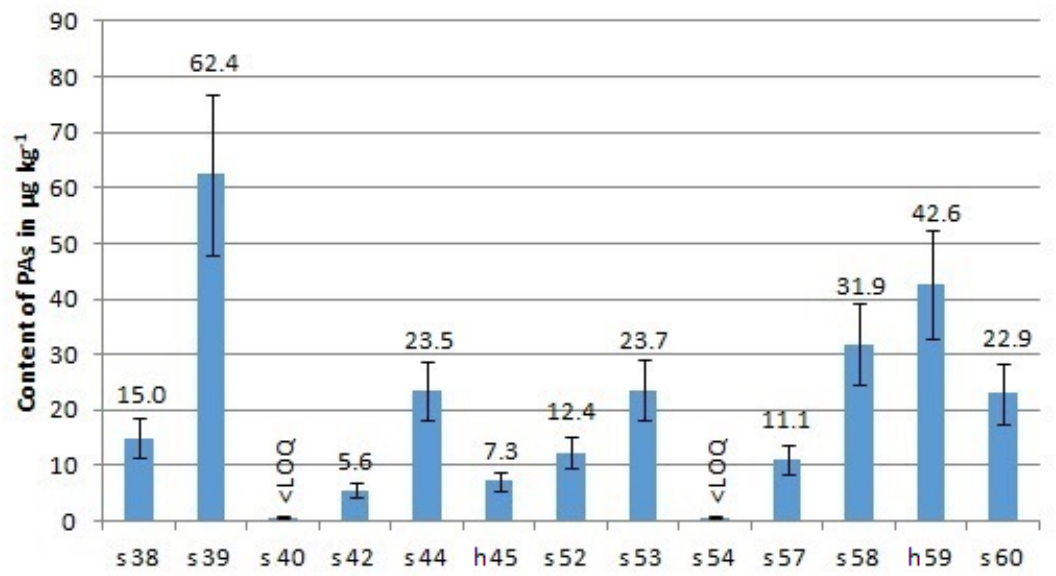

Fig. 3. The content of PAs in positive feed samples. Silage (s), hay (h) 
Only one sample (silage) showed a high total PAs content of $62.4 \mu \mathrm{g} \mathrm{kg}^{-1}$. The highest determined PAs concentration in hay samples was $42.6 \mu \mathrm{g} \mathrm{kg}^{-1}$. The overall detected PAs concentrations ranged from 5.6 to $62.4 \mu \mathrm{g} \mathrm{kg}^{-1}$, and the average content and median for positive samples were $20.1 \mu \mathrm{g} \mathrm{kg}^{-1}$ and $14.9 \mu \mathrm{g} \mathrm{kg}^{-1}$, respectively. When all samples were included in the calculation, the average PAs concentration was $8.11 \mu \mathrm{g} \mathrm{kg}^{-1}$.

Six samples contained more than one monitored alkaloid. The most abundant PAs were lycopsamine and intermedine. Lycopsamine was present in $61.5 \%$ of all positive samples, with the determined concentrations ranging from 5.5 to $31.8 \mu \mathrm{g} \mathrm{kg}^{-1}$. The concentration range of intermedine was $<\mathrm{LOQ}-$ $55.1 \mu \mathrm{g} \mathrm{kg}^{-1}$ and the alkaloid was present in $46.2 \%$ of all positive samples. Senecionine ( $\left.<\mathrm{LOQ}-14.9 \mu \mathrm{g} \mathrm{kg}^{-1}\right)$, retrorsine $\left(<\mathrm{LOQ}-7.2 \mu \mathrm{g} \mathrm{kg}^{-1}\right)$, and senkirkine $\left(6.1 \mu \mathrm{g} \mathrm{kg}^{-1}\right)$ were among other detected alkaloids.

\section{Discussion}

The analysis of plant materials is still problematic even if state-of-the-art analytical equipment is used. PAs are of purely natural origin in contrast to antibiotics or pesticides (11), and are coextracted with many other natural compounds. The variation in composition of feed materials and trace concentrations of PAs pose analytical challenges regarding sample preparation and instrumental analysis. Therefore, an appropriate cleaning strategy seems to be one of the most important steps in the sample preparation process.

In a previously conducted study GC-MS was used for the determination of PAs in feeds (21). However, LC-MS technique has one advantage over GC-MS: it provides structural information and therefore alkaloids contributing to the overall contamination can be identified. Moreover, the procedure of sample preparation can be simplified as it does not require PAs reduction to the necine base backbone structures and a subsequent derivatisation step.

Previously hydrochloric acid was chosen as the most effective solvent for PAs extraction from feed matrix. However, in the case of liquid chromatography, better results, especially when it comes to the purity of the chromatograms, were obtained with sulphuric acid. Nevertheless, the recoveries, as well as the conversion of $N$-oxides into their free base forms were very comparable with the use of both acids, as it was also found before (21).

In contrast to honey analysis, in the case of feed and other plant materials, there is no single dominant method of sample preparation and extract purification. Bolechová et al. (2) applied the QuEChERS method and extraction with acetonitrile and $0.1 \%$ formic acid in water. Huybrechts and Callebaut (20) extracted the PAs with an aqueous solution of $\mathrm{HCl}$ with the addition of $\mathrm{NaCl}$ and analysed the extracts without further purification. Mulder et al. (25) used 2\% formic acid for the extraction of PAs from feeds followed by purification on Strata X cartridges with methanol used for PAs elution. Gottschalk et al. (18) also used 2\% formic acid for PAs isolation, however, for the purification cation exchange cartridges and PAs elution with 5\% ammonia in methanol were applied. For extraction of PAs from herbal products and teas Crammer et al. (6), Bodi et al. (1), and Schulz et al. (29) utilised $0.05 \mathrm{M}$ sulphuric acid and purification with $\mathrm{C} 18$ or cation exchange cartridges. For the PAs elution methanol or ammonia in methanol were selected. In all cases LC-MS/MS was used for the instrumental analysis. However, none of the approaches described above were useful for the LC-MS analysis. The obtained eluates were not properly purified and many other compounds interfered with the target PAs. In fact, the detection of PAs at the concentration of $20 \mu \mathrm{g} \mathrm{kg}^{-1}$ was impossible when $3 \%$ ammonia in methanol was used as an eluent in combination with an Strata SCX cartridge (Fig. 2a). For this reason a new protocol for sample purification had to be developed.

In the previously conducted studies, a new elution mixture consisting of ethyl acetate, methanol, ammonia and triethylamine $(8: 2: 0.1: 0.1 \mathrm{v} / \mathrm{v})$ was developed to improve the sufficiency of the SPE clean-up of honey matrix (22).

This elution mixture was also tested in the SPE purification of feed extracts, and it gave much better results than $3 \%$ of ammonia in methanol. However, to obtain the most efficient results, a different combination of solvents (ethyl acetate, methanol, ammonia, triethylamine, and acetonitrile) and different cation exchange cartridges (MCX, Strata SCX, PCX, Strata XC, and HF) were also evaluated.

The best purification effect was obtained for the Strata SCX cartridge; however, when the solvent combination was regarded, the best results were yielded by the mixture consisting of ethyl acetate, methanol, acetonitrile, ammonia, and triethylamine (8:1:1:0.1:0.1, $\mathrm{v} / \mathrm{v})$. The recovery rates of all target alkaloids were also at satisfactory levels. Application of this new elution mixture had crucial importance especially in the case of identification and quantification of the low concentration of monitored PAs in feed materials. In comparison to elution with $3 \%$ ammonia in methanol, the quantification of PAs at the level of $5 \mu \mathrm{g} \mathrm{kg}^{-1}$ could be carried out easily (Fig. 2b). Consequently, it positively influenced the sensitivity of the LC-MS method and could be compared with protocols based on the LC-MS/MS technique $(2,25)$.

The mobile phase has to be optimised to obtain the best separation of the analysed compounds. Formic acid of different concentrations, in several cases in combination with ammonium formate $(1,6,18,29)$, was used as the most popular modifier of water and organic mobile phases $(4,11,17,23)$. In all published methodologies acetonitrile or methanol was used as the organic phase. However, proposed mobile phases did 
not always provide good separation of all alkaloids, especially when it came to enantiomeric structures such as intermedine and lycopsamine that usually are coeluted. In our method, the mixture of methanol and acetonitrile $(1: 1, \mathrm{v} / \mathrm{v})$ was used as the organic phase. This combination of the organic solvents provided good separation of all analysed alkaloids, including the enantiomeric structures.

Several reversed phase C18 columns were tested during optimisation of the separation of the analysed PAs. The best results as far as the separation and peak shape are concerned were obtained with a Gemini NX$\mathrm{C} 18,150 \mathrm{~mm} \times 4.6 \mathrm{~mm}, 3 \mu \mathrm{m}$ column and a mobile phase consisting of $0.2 \%$ formic acid in water (A) and a mixture of methanol and acetonitrile $(1: 1, \mathrm{v} / \mathrm{v})(\mathrm{B})$.

PAs have easily ionisable nitrogen in the ring system and the protonation of PAs molecules can be achieved (7), that is why ESI in a positive ionisation mode was chosen for the analysis. To optimise all parameters affecting ESI source operation, flow injection analysis (FIA) was used.

The method was successfully validated according to the SANTE/11945/2015 recommendations (28). Quantification was based on matrix calibration curves. The method showed adequate linearity in a concentration range $0-100 \mu \mathrm{g} \mathrm{kg}^{-1}$ with determination coefficient $\mathrm{R}^{2}>0.99$ for all analysed alkaloids. All determined parameters met the SANTE document criteria as recovery was in the range of $70 \%-120 \%$ and relative standard deviations of repeatability and reproducibility were $\leq 20 \%$. The obtained recovery values for the target PAs were in the range $84.1 \%-112.9 \%$ and were slightly higher than values reported by Bolechová et al. (2), who achieved recoveries in the range of $72 \%-98 \%$, and Gottschalk et al. (18), who found recoveries in the range of $69 \%-104 \%$. The method proved to be selective, as no interfering peaks were determined in the retention times assigned to the particular alkaloid. It can also be affirmed that the method is robust to slight changes in selected performance parameters.

Feeds are considered difficult and complex materials containing a lot of compounds which might interfere and cause both signal suppression and signal enhancement at MS detection. A matrix effect could be noticed in the case of alkaloids such as jacobine or senkirkine; however, the values are still in the acceptable range. According to the PA stability results, the purified extracts can be stored for about a week, which is in line with previously obtained results for extracts prepared for GC-MS analysis (21).

Plant material is a very difficult matrix, and often methods based on LC-MS/MS have higher LOQ values for the same alkaloids when analysed in plant material than matrices such as honey, meat, or eggs $(1,26)$. For the developed method the LOQ was established at $5 \mu \mathrm{g} \mathrm{kg}^{-1}$ for the individual alkaloid, as it was the lowest validation level. This LOQ value is comparable with, or even lower than LOQs reported for methods based on LC-MS/MS methodology that were used for determination of PAs in feeds $(2,25)$ or in other plant materials such as herbal teas (29).

The developed method was applied to the analysis of 32 feed samples. Among detected alkaloids, lycopsamine and intermedine were the most abundant compounds, followed by senecionine and retrorsine. This is in line with Huybrechts and Callebaut (20), who also reported that lycopsamine and intermedine were the most frequently detected alkaloids in feed samples with a lower contribution of senecionine and retrorsine. Also, Gottschalk et al. (18) reported the presence of intermedine, lycopsamine and senecionine in analysed silage samples. Bolechová et al. (2) determined retrorsine and senecionine in feed for chickens and senkirkine in silage samples. However, in contrast to other authors reporting PAs contamination in feed materials $(2,20,25)$, seneciphylline was not detected in any of the analysed materials. This may be due to the difference in the plant species, which was a source of contamination, as well as the variability of the composition and concentration of alkaloids in the same plant species, which strongly depends on the environmental conditions prevailing in a particular year, growth stage, part of the plant, and geographical origin.

Determined concentrations can be regarded as low and are consistent with results reported in the previously conducted study where a GC-MS technique was used for PAs determination in feed materials (21). The determined levels are also in agreement with the PAs contamination results of feeds of European origin reported by Mulder et al. (25), Bolechová et al. (2), Huybrechts and Callebaut (20), and Gottschalk et al. (18). Mean values of PAs concentrations in silage reported by the authors were in the range of 4.8-25.7 $\mu \mathrm{g} \mathrm{kg}^{-1}$. In our study the average content of PAs in positive samples was $20.1 \mu \mathrm{g} \mathrm{kg}^{-1}$. Mulder et al. (25) and Gottschalk et al. (30) reported $30 \mu \mathrm{g} \mathrm{kg}^{-1}$ as the highest PAs concentration detected in silage samples. In our study, the highest PAs content was determined at the level of $62.4 \mu \mathrm{g} \mathrm{kg}^{-1}$, which is higher but is still comparable with other authors' results.

Hoogenboom et al. (19) studied the transfer of PAs from feed to milk in dairy cows. The transfer rate was about $0.1 \%$ of the overall daily dose of PAs. The authors showed that the rate of PAs transferred to milk depended on the type of alkaloid. $N$-oxides were not detected in milk even though they made a large contribution to the content of PAs in contaminated feed. However, the highest transfer rate was observed for jacoline, even though it was not the main contributor to the overall content of PAs in feed (19, 27). This was also confirmed by Mulder et al. (27), who studied the transfer of PAs to eggs and meat in laying hens. The author found that the transfer rates were relatively low, ranging from $0.02 \%$ to $0.23 \%$ and also depended on the PAs composition of the plant containing the alkaloids (27). Considering the transfer 
rates and the highest content of PAs determined in our study, it can be concluded that the detected concentrations should not pose the risk of alkaloid transfer into food such as milk or meat.

Regarding acute poisoning, it can also be stated that the determined PAs concentrations should not pose a risk to the animals. The reported cumulative lethal dose for cows is $2.5 \mathrm{mg}$ PAs kg-1 body weight per day (which translates to $1,500 \mathrm{mg}$ for a cow of $600 \mathrm{~kg}$ ) during exposure for 18 days $(18,30)$. In our study, the highest detected concentration was $62.4 \mu \mathrm{g} \mathrm{kg}^{-1}$, which would be only $0.06 \%$ of the lethal dose in the case of $15 \mathrm{~kg}$ of silage consumption. However, the detected concentrations, if consumed in the long term, especially in combination with other toxins, may adversely affect animals.

In conclusion, the EFSA has recommended the development of sensitive and selective methods suitable for determination of PAs in feeds, and the presented method fulfils the Agency's requirements of selectivity and sensitivity. Application of the new elution mixture consisting of ethyl acetate, methanol, acetonitrile, ammonia, and triethylamine significantly improved the purification effect and markedly improved the sensitivity of the method. Validation according to SANTE/11945/2015 proved the method's reliability. The developed method was applied to the analysis of 32 feed samples, of which almost $41 \%$ were positive for at least one of the PAs. Detected PAs concentrations were relatively low and should not be of concern as a cause of acute poisoning of animals or the transfer of PAs to food of animal origin; however, in the long term consumption they may affect the health of animals.

Conflict of Interests Statement: The authors declare that there is no conflict of interests regarding the publication of this article.

Financial Disclosure Statement: The research was financed from the Institute's statutory sources.

Animal Rights Statement: None required.

Acknowledgements: The authors are grateful to Elżbieta Kukier, Magdalena Goldsztejn, and Katarzyna Pietruszka for sharing the feed samples.

\section{References}

1. Bodi D., Ronczka S., Gottschalk C., Behr N., Skibba A., Wagner M., Lahrssen-Wiederholt M., Preiss-Weigert A., These A.: Determination of pyrrolizidine alkaloids in tea, herbal drugs, and honey. Food Addit Contam Part A 2014, 31, 1886-1895.

2. Bolechová M., Čáslavský J., Pospíchalová M., Kosubová P.: UPLC-MS/MS method for determination of selected pyrrolizidine alkaloids in feed. Food Chem 2015, 170, 265-270.
3. Boppré M.: The ecological context of pyrrolizidine alkaloids in food, feed, and forage: an overview. Food Addit Contam Part A 2011, 28, 260-281.

4. Boppré M., Colegate S.M., Edgar J.A.: Pyrrolizidine alkaloids of Echium vulgare honey found in pure pollen. J Agric Food Chem 2005, 53, 594-600.

5. Chen L., Mulder P.P.J., Louisse J., Peijnenburg A., Wesseling S. Rietjens I.M.C.M.: Risk assessment for pyrrolizidine alkaloids detection in (herbal) teas and plant supplements. Regul Toxicol Pharm 2017, 86, 292-302.

6. Cramer L., Schiebel H.M., Ernst L., Beuerle T.: Pyrrolizidine alkaloids in the food chain: development, validation, and application of a new HPLC-ESI-MS/MS sum parameter method. J Agric Food Chem 2013, 61, 11382-11391.

7. Crews C., Berthiller F., Krska R.: Update on analytical methods for toxic pyrrolizidine alkaloids. Anal Bioanal Chem 2010, 396, 327-338.

8. de Nijs M., Mulder P.P.J., Klijnstra M.D., Driehuis F., Hoogenboom R.L.A.P.: Fate of pyrrolizidine alkaloids during processing of milk of cows treated with ragwort. Food Addit Contam Part A 2017, 34, 2212-2219.

9. Danezis G.P., Anagnostopoulos C.J., Liapis K., Koupparis M.A.: Multi-residue analysis of pesticides, plant hormones, veterinary drugs, and mycotoxins using HILIC chromatography MS/MS in various food matrices. Anal Chimica Acta 2016, 942, 121-138.

10. Dreger M., Stanislawska M., Krajewska-Patan A., Mielcarek S., Mikolajczak P.L., Buchwald W.: Pyrrolizidine alkaloids chemistry, biosynthesis, pathway, toxicity, safety, and perspectives of medicinal usage. Herba Pol 2009, 55, 127-147.

11. Dübecke A., Beckh G., Lüllmann C.: Pyrrolizidine alkaloids in honey and bee pollen. Food Addit Contam Part A 2011, 28, 348-358.

12. Edgar J.A., Colegate S.M., Boppre M., Molyneux R.J.: Pyrrolizidine alkaloids in food: a spectrum of potential health consequences. Food Addit Contam Part A 2011, 28, 308-324.

13. Edgar J.A., Molyneux R.J., Colegate S.M.: Pyrrolizidine alkaloids: potential role in the aetiology of cancers, pulmonary hypertension, congenital anomalies, and liver disease. Chem Res Toxicol 2015, 28, 4-20.

14. Edgar J.A., Roeder E., Molyneaux R.J.: Honey from plants containing pyrrolizidine alkaloids: a potential threat to health. J Agric Food Chem 2002, 50, 2719-2730.

15. European Food Safety Authority, EFSA Panel on Contaminants in the Food Chain (CONTAM). Scientific opinion on pyrrolizidine alkaloids in food and feed. EFSA J 2011, 9:2406, $1-134$.

16. Fu P.P., Xia Q., Lin G., Chou M.W.: Pyrrolizidine alkaloidsgenotoxicity, metabolism enzymes, metabolic activation, and mechanisms. Drug Metab Rev 2004, 36, 1-55.

17. Griffin C.T., Danaher M., Elliott C.T., Kennedy D.G., Furey A.: Detection of pyrrolizidine alkaloids in commercial honey using liquid chromatography-ion trap mass spectrometry. Food Chem 2013, 136, 1577-1583.

18. Gottschalk C., Ronczka S., Preiß-Weigert A., Ostertag J., Klaffke H., Schafft H., Lahrssen-Wiederholt M.: Pyrrolizidine alkaloids in natural and experimental grass silages and implications for feed safety. Anim Feed Sci Tech 2015, 207, 253-261.

19. Hoogenboom L.A., Mulder P.P., Zeilmaker M.J., van den Top H.J., Remmelink G.J., Brandon E.F., Klijnstra M., Meijer G.A., Schothorst R., Van Egmond H.P.: Carry-over of pyrrolizidine alkaloids from feed to milk in dairy cows. Food Addit Contam Part A 2011, 28, 359-372.

20. Huybrechts B., Callebaut A.: Pyrrolizidine alkaloids in food and feed on the Belgian market. Food Addit Contam Part A 2015, 32, 1939-1951.

21. Kowalczyk E., Kwiatek K.: Determination of pyrrolizidine alkaloids in selected feed materials with gas chromatographymass spectrometry. Food Addit Contam Part A 2017, 34, $853-863$. 
22. Kowalczyk E., Sieradzki Z., Kwiatek K.: Determination of pyrrolizidine alkaloids in honey with sensitive gas chromatography-mass spectrometry method. Food Anal Methods 2018, 11, 1345-1355.

23. Martinello M., Cristofoli C., Gallina A., Mutinelli F.: Easy and rapid method for the quantitative determination of pyrrolizidine alkaloids in honey by ultra performance liquid chromatographymass spectrometry: An evaluation in commercial honey. Food Control 2014, 37, 146-152.

24. Merz K.H., Schrenk D.: Interim relative potency factors for the toxicological risk assessment of pyrrolizidine alkaloids in food and herbal medicines. Toxicol Lett 2016, 263, 44-57.

25. Mulder P.P.J., Beumer B., Oosterink E., de Jong J.: Dutch survey pyrrolizidine alkaloids in animal forage, RIKILT Report No 2009.018, Wegeningen 2009.

26. Mulder P.P.J., López Sánchez P., These A., Preiss-Weigert A., Castellari M.: Occurrence of pyrrolizidine alkaloids in food. 2015, EFSA:EN-859, 1-116.
27. Mulder P.P.J., de Witte S.L., Stoopen G.M., van der Meulen J., van Wikselaar P.G., Gruys E., Groot M.J., Hoogenboom R.L: Transfer of pyrrolizidine alkaloids from various herbs to eggs and meat in laying hens. Food Addit Contam Part A 2016, 33, 1826-1839.

28. SANTE/11945/2015 Guidance document on analytical quality control and method validation procedures for pesticides residues analysis in food and feed. Supersedes SANCO/12571/2013, Implemented by 01/01/2016, 1-42.

29. Schulz M., Meins J., Diemert S., Zagermann-Muncke P., Goebelm R., Schrenkm D., Schubert-Zsilaveczm M., AbdelTawabm M.: Detection of pyrrolizidine alkaloids in German licensed herbal medicinal teas. Phytomedicine 2015, 22, 648-656.

30. Stegelmeier B.L., Edgar J.A., Colegate S.M., Gardner D.R., Schoch T.K., Coulombe R.A., Molyneux R.J.: Pyrrolizidine alkaloid plants, metabolism and toxicity. J Nat Toxins 1999, 8, 95-116. 